\title{
INTERPOLATION BETWEEN SOBOLEV SPACES IN LIPSCHITZ DOMAINS WITH AN APPLICATION TO MULTIGRID THEORY
}

\author{
JAMES H. BRAMBLE
}

\begin{abstract}
In this paper we describe an interpolation result for the Sobolev spaces $H_{0}^{s}(\Omega)$ where $\Omega$ is a bounded domain with a Lipschitz boundary. This result is applied to derive discrete norm estimates related to multilevel preconditioners and multigrid methods in the finite element method. The estimates are valid for operators of order $2 m$ with Dirichlet boundary conditions.
\end{abstract}

\section{INTRODUCTION}

Let $\Omega$ be a bounded domain with a Lipschitz boundary. In this note we show that the Sobolev space $H_{0}^{r}(\Omega)$, for $n$ an integer and $r=1, \ldots, n-1$, is the same space as that obtained by interpolation between the spaces $H_{0}^{n}(\Omega)$ and $L_{2}(\Omega)$. The interpolation spaces are defined by means of the real method of interpolation of Lions and Peetre [9]. This is proved in [8] when the boundary of $\Omega$ is smooth. For plane domains with piecewise smooth boundaries it was proved by Zolesio in [11]. The resulting space $\left[H_{0}^{n}(\Omega), L_{2}(\Omega)\right]_{r / n}$ has a different Hilbert space structure from the usual space $H_{0}^{r}(\Omega)$. For $n=2 m$ and $r=m$ the regularity properties associated with the elliptic pseudodifferential operator defined by the corresponding inner product are generally better than those of the standard $m$ th power of the Laplacian with Dirichlet boundary conditions. This will be used to prove some estimates connected with multilevel finite element methods.

In order to motivate the discussion, we shall consider the following. Employing the notation in [1], let $M$ be a finite-dimensional space equipped with an inner product $(\cdot, \cdot)$ with corresponding norm $\|\cdot\|$. Let $A(\cdot, \cdot)$ be a symmetric bilinear form on $M$. Now set $M=M_{J}$ and suppose that we have subspaces $M_{k}$ with

$$
M_{1} \subset M_{2} \subset \cdots \subset M_{J} \equiv M .
$$

Received by the editor September 16, 1994.

1991 Mathematics Subject Classification. Primary 65N30; Secondary 65F10.

This manuscript has been authored under contract number DE-AC02-76CH00016 with the U.S. Department of Energy. Accordingly, the U.S. Government retains a nonexclusive, royalty-free license to publish or reproduce the published form of this contribution, or allow others to do so, for U.S. Government purposes. This work was also supported in part under the National Science Foundation Grant No. DMS-9007185 and by the U.S. Army Research Office through the Mathematical Sciences Institute, Cornell University, Contract No. DAAL03-91-C-0027.

(C) 1995 American Mathematical Society 
The linear operator $A_{k}: M_{k} \rightarrow M_{k}$ is defined by

$$
\left(A_{k} \psi, \theta\right)=A(\psi, \theta) \quad \text { for all } \psi, \theta \in M_{k} .
$$

Furthermore, the projectors $P_{k}: M_{J} \rightarrow M_{k}$ and $Q_{k}: M_{J} \rightarrow M_{k}$ are defined by

$$
A\left(P_{k} u, v\right)=A(u, v)
$$

and

$$
\left(Q_{k} u, v\right)=(u, v),
$$

for all $u \in M_{J}$ and all $v \in M_{k}$. In the problem considered in $\S 4$ of this paper, $Q_{k}$ is the $L_{2}$-projection and $P_{k}$ is the so-called elliptic projection. Let $\lambda_{k}$ be the largest eigenvalue of $A_{k}$. The condition

$$
\sum_{k=1}^{J} \lambda_{k}\left\|\left(Q_{k}-Q_{k-1}\right)\right\|^{2} \leq C A(v, v) \text { for all } v \in M_{J},
$$

with the constant $C$ independent of $J$, plays a central role in both the additive and muliplicative multilevel theory, cf. [1]. This was first pointed out in [3], where the additive multilevel preconditioner was introduced. It was proved there only in the case of "full elliptic regularity". This regularity property does not hold in the case of second-order elliptic operators on nonconvex polygonal plane domains or for higher-order operators even on convex nonsmooth domains. Inequality (1.1) was shown to hold much more generally in the second-order case by Oswald [10] using Besov space arguments. A different proof based on the knowledge of a modest amount of elliptic regularity was later provided in [2]. Here we show that for any Lipschitz domain we can define an operator in the equivalence class for which full elliptic regularity holds, thus proving (1.1) in general in the second-order case. This is done by using the above interpolation result.

Remark 1 . In the above discussion and in what follows, the spaces $H_{0}^{s}(\Omega)$ may be replaced by $H^{s}(\Omega)$, thus allowing us to consider also the cases in which the boundary conditions are natural. In this case the development is either completely analogous or simpler. Thus, we will consider only the case of the Dirichlet problem.

\section{INTERPOLATION BETWEEN THE SPACES $H_{0}^{n}(\Omega)$ AND $L^{2}(\Omega)$}

We recall here the real method of interpolation. Let $B_{0}$ and $B_{1}$ be two Banach spaces with $B_{1}$ continuously embedded and dense in $B_{0}$. An intermediate space $B$ is any subspace of $B_{0}$ satisfying

$$
B_{1} \subset B \subset B_{0} \text {. }
$$

Real Method of Interpolation. We shall define for $0<s<1$ a scale of spaces $\left[B_{1}, B_{0}\right]_{s}$ with

$$
B_{1} \subset\left[B_{1}, B_{0}\right]_{s} \subset B_{0} .
$$

Define for each $t>0$ and $u \in B_{1}$

$$
K(t, u)=\inf _{u_{0}+u_{1}=u}\left(\left\|u_{0}\right\|_{B_{0}}^{2}+t^{2}\left\|u_{1}\right\|_{B_{1}}^{2}\right)^{1 / 2},
$$

where $u_{0} \in B_{0}, u_{1} \in B_{1}$ and a standard notation is used to denote the norms. 
For $0<s<1$ define the quantity

$$
\|u\|_{\left[B_{1}, B_{0}\right]_{s}}=\left(\int_{0}^{\infty} t^{-2 s} K^{2}(t, u) \frac{d t}{t}\right)^{1 / 2},
$$

which is a norm and hence defines a Banach space $\left[B_{1}, B_{0}\right]_{s}$ which is intermediate to $B_{1}$ and $B_{0}$. It is elementary to see that if $B_{1}$ and $B_{0}$ are Hilbert spaces then this norm satisfies the parallelogram law and therefore induces a Hilbert space structure.

We now prove a result for Sobolev spaces on domains with Lipschitz boundaries. As previously noted, such a result may be found in [8] in the case of smooth boundaries and for plane domains with piecewise smooth boundaries in [11]. As usual, $c$ and $C$ will be used to denote generic constants, not necessarily the same in any two places.

Theorem 2.1. Let $\Omega$ be a bounded domain with a Lipschitz boundary. Then, for $n$ an integer and $r=1, \ldots, n-1$,

$$
\left[H_{0}^{n}(\Omega), L_{2}(\Omega)\right]_{r / n}=H_{0}^{r}(\Omega)
$$

with equivalent norms.

Proof. For $u$ defined in $\Omega$, let $\tilde{E}$ denote extension by zero to $R^{d}$.

Define

$$
\tilde{H}^{r}(\Omega):=\left\{u \in H^{r}(\Omega) \mid \quad \tilde{E} u \in \dot{H}^{r}\left(R^{d}\right)\right\}
$$

and set

$$
\|u\|_{\tilde{H}^{r}(\Omega)}=\|\tilde{E} u\|_{H^{r}\left(R^{d}\right)} .
$$

Recall that $H_{0}^{r}(\Omega)$ is the completion of $\mathscr{D}(\Omega)$ under the norm $\|\cdot\|_{H^{r}(\Omega)}$. By Theorem 1.4.2.2 in [7],

$$
\tilde{H}^{r}(\Omega)=H_{0}^{r}(\Omega) .
$$

We will first show that

$$
\left[H_{0}^{n}(\Omega), L_{2}(\Omega)\right]_{r / n} \subset H_{0}^{r}(\Omega) .
$$

To do this, let $u \in H_{0}^{j}(\Omega)$, for $j=0$ or $j=2 m$. Since $\tilde{H}^{j}(\Omega)=H_{0}^{j}(\Omega)$, we have that

$$
\|\tilde{E} u\|_{H^{j}\left(R^{d}\right)}=\|u\|_{\tilde{H}^{j}(\Omega)} \leq C\|u\|_{H_{0}^{j}(\Omega)} .
$$

Hence by interpolation of operators, since

$$
\left[H^{n}\left(R^{d}\right), L_{2}\left(R^{d}\right)\right]_{r / n}=H^{r}\left(R^{d}\right),
$$

we have that

$$
c\|u\|_{H_{0}^{r}(\Omega)} \leq\|u\|_{\tilde{H}^{r}(\Omega)}=\|\tilde{E} u\|_{H^{r}\left(R^{d}\right)} \leq C\|u\|_{\left[H_{0}^{n}(\Omega), L_{2}(\Omega)\right]_{r / n}} .
$$

This shows that $\left[H_{0}^{n}(\Omega), L_{2}(\Omega)\right]_{r / n} \subset H_{0}^{r}(\Omega)$.

For the opposite inclusion, we construct an operator $R: H^{j}\left(R^{d}\right) \mapsto H_{0}^{j}(\Omega)$ as follows. Let $\mathscr{B}$ be an open ball such that $\bar{\Omega} \subset \mathscr{B}$ and let $E$ be a continuous extension operator from $H^{j}(\mathscr{B} \backslash \Omega)$ to $H^{j}\left(R^{d}\right)$ for $0 \leq j \leq n$. We can do this by virtue of the fact that $\Omega$ has a Lipschitz boundary. Now define $R$ by

$$
R u=u-E u
$$


restricted to $\Omega$. Let $\psi \in C_{0}^{\infty}(\mathscr{B})$ be a cutoff function which is equal to one on $\Omega$. Then the function $\psi(u-E u)$, defined on all of $R^{d}$, is in $H^{j}\left(R^{d}\right)$ and

$$
\psi(u-E u)=\tilde{E} R u \text {. }
$$

Hence, $R u \in \tilde{H}^{j}(\Omega)=H_{0}^{j}(\Omega)$. Now

$$
\begin{aligned}
\|R u\|_{H_{0}^{j}(\Omega)} & \leq C\left(\|u\|_{H^{j}(\Omega)}+\|E u\|_{H^{j}(\Omega)}\right) \\
& \leq C\|u\|_{H^{j}\left(R^{d}\right)},
\end{aligned}
$$

for $j=0$ or $j=n$. Hence,

$$
\|R u\|_{\left[H_{0}^{n}(\Omega), L_{2}(\Omega)\right]_{r / n}} \leq C\|u\|_{H^{r}\left(R^{d}\right)} .
$$

But if $u \in H_{0}^{r}(\Omega)$, then $\tilde{E} u \in H^{r}\left(R^{d}\right)$ and $R \tilde{E} u=u$. Hence,

$$
\|u\|_{\left[H_{0}^{n}(\Omega), L_{2}(\Omega)\right]_{r / n}}=\|R \tilde{E} u\|_{\left[H_{0}^{n}(\Omega), L_{2}(\Omega)\right]_{r / n}} \leq C\|\tilde{E} u\|_{H^{r}\left(R^{d}\right)} \leq C\|u\|_{H_{0}^{r}(\Omega)} .
$$

Hence it follows that $H_{0}^{r}(\Omega) \subset\left[H_{0}^{n}(\Omega), L_{2}(\Omega)\right]_{r / n}$. This proves the theorem.

Note that $H_{0}^{r}(\Omega)$ and $\left[H_{0}^{n}(\Omega), L_{2}(\Omega)\right]_{r / n}$ may be different Hilbert spaces.

\section{BILINEAR FORMS}

It is well known that for $k<l$ the space $H_{0}^{l}(\Omega)$ is compactly imbedded in $H_{0}^{k}(\Omega)$ (cf. [7]). Denoting the usual inner product on $H_{0}^{2 m}(\Omega)$ by $(\cdot, \cdot)_{2 m}$, the operator $T: L_{2}(\Omega) \mapsto H_{0}^{2 m}(\Omega)$ defined by

$$
(T u, \phi)_{2 m}=(u, \phi) \quad \text { for all } \phi \in H_{0}^{2 m}(\Omega)
$$

is positive definite and compact, with positive eigenvalues $\left\{\Lambda_{i}^{-1}\right\}$. The corresponding eigenvectors $\left\{\phi_{i}\right\}$ are complete in $L_{2}(\Omega)$ and satisfy $\Lambda_{i} T \phi_{i}=\phi_{i}$. It is easy to see that for $u=\sum_{i=1}^{\infty} c_{i} \phi_{i}$, the norm on $H_{0}^{2 m}(\Omega)$ is given by

$$
\|u\|_{H_{0}^{2 m}(\Omega)}=\left(\sum_{i=1}^{\infty} \Lambda_{i} c_{i}^{2}\right)^{1 / 2} .
$$

The space $\hat{H}_{0}^{m}(\Omega)=\left[H_{0}^{2 m}(\Omega), L_{2}(\Omega)\right]_{1 / 2}$ has the norm

$$
\|u\|_{\hat{H}_{0}^{m}(\Omega)}=\left(\sum_{i=1}^{\infty} \Lambda_{i}^{1 / 2} c_{i}^{2}\right)^{1 / 2},
$$

which induces the inner product, say $\mathscr{A}(\cdot, \cdot)$, with

$$
\mathscr{A}(u, u)=\sum_{i=1}^{\infty} \Lambda_{i}^{1 / 2} c_{i}^{2}
$$

This norm is equivalent to the standard norm by Theorem 2.1. The spaces $\hat{H}_{0}^{m+s}(\Omega)=\left[H_{0}^{2 m}(\Omega), L_{2}(\Omega)\right]_{1 / 2+s / 2 m}$ and $\hat{H}_{0}^{m-s}(\Omega)=\left[H_{0}^{2 m}(\Omega), L_{2}(\Omega)\right]_{1 / 2-s / 2 m}$, for $0 \leq s \leq m$, have the norms

$$
\|u\|_{\hat{H}_{0}^{m+s}(\Omega)}=\left(\sum_{i=1}^{\infty} \Lambda_{i}^{1 / 2+s / 2 m} c_{i}^{2}\right)^{1 / 2}
$$

and

$$
\|u\|_{\hat{H}_{0}^{m-s}(\Omega)}=\left(\sum_{i=1}^{\infty} \Lambda_{i}^{1 / 2-s / 2 m} c_{i}^{2}\right)^{1 / 2}
$$

The following is now obvious. 
Theorem 3.1. Let $\Omega$ be a bounded domain with a Lipschitz boundary. Then for $u \in H_{0}^{m-s}(\Omega)$ and $\phi \in H_{0}^{m+s}(\Omega)$,

$$
\mathscr{A}(u, \phi)=\sum_{i=1}^{\infty} \Lambda_{i}^{1 / 2}\left(u, \phi_{i}\right)\left(\phi, \phi_{i}\right)
$$

is well defined and

$$
\|u\|_{\hat{H}_{0}^{m-s}(\Omega)}=\sup _{0 \neq \phi \in \hat{H}_{0}^{m+s}(\Omega)} \frac{\mathscr{A}(u, \phi)}{\|\phi\|_{\hat{H}_{0}^{m+s}(\Omega)}} .
$$

\section{REGULARITY FOR THE FORM $\mathscr{A}$}

We shall consider the solution $u \in H_{0}^{m}(\Omega)$ of the problem

$$
\mathscr{A}(u, \phi)=(f, \phi) \text { for all } \phi \in H_{0}^{m}(\Omega),
$$

where $f \in L^{2}(\Omega)$. The following theorem is easy.

Theorem 4.1. Let $u$ be a solution of (4.1) with $f \in L^{2}(\Omega)$. Then $u \in H_{0}^{2 m}(\Omega)$ and

$$
\|u\|_{H_{0}^{2 m}(\Omega)} \leq\|f\|_{L^{2}(\Omega)} \text {. }
$$

Proof. Choose $\phi=\sum_{i=1}^{n} \Lambda_{i}^{1 / 2}\left(u, \phi_{i}\right) \phi_{i}$ in (4.1) and, after applying the CauchySchwarz inequality, let $n$ tend to infinity.

Let $\left\{S_{h}\right\}, h>0$, be a family of subspaces of $H_{0}^{m}(\Omega)$ with the following approximation property. There exists a constant $C>0$ such that for any $v \in H_{0}^{m+s}(\Omega)$

$$
\inf _{\chi \in S_{h}}\|v-\chi\|_{H_{0}^{m}(\Omega)} \leq C h^{s}\|v\|_{H_{0}^{m+s}(\Omega)},
$$

for some $s$ with $0<s \leq m$. For many examples of such spaces see [5] or [6].

Now the discrete solution of (4.1) in $S_{h}$ is the unique solution $U \in S_{h}$ such that

$$
\mathscr{A}(U, \phi)=(f, \phi) \text { for all } \phi \in S_{h} .
$$

Define the orthogonal projection operator $P_{h}: H_{0}^{m}(\Omega) \mapsto S_{h}$ by means of

$$
\mathscr{A}\left(P_{h} u, \phi\right)=\mathscr{A}(u, \phi) \quad \text { for all } \phi \in S_{h} \text {. }
$$

Since $\mathscr{A}(\cdot, \cdot)$ defines a norm on $H_{0}^{m}(\Omega)$, we have, using (4.2),

$$
\begin{aligned}
\left\|u-P_{h} u\right\|_{H_{0}^{m}(\Omega)} & \leq C \inf _{\chi \in S_{h}}\|v-\chi\|_{H_{0}^{m}(\Omega)} \\
& \leq C h^{s}\|u\|_{H_{0}^{m+s}(\Omega)} \quad \text { for all } u \in H_{0}^{m+s}(\Omega) .
\end{aligned}
$$

For this projector we now have the following "regularity pickup".

Theorem 4.2. Let $u \in H_{0}^{m+s}(\Omega)$. If (4.2) is satisfied, then

$$
\left\|u-P_{h} u\right\|_{H_{0}^{m-s}(\Omega)} \leq C h^{s}\|u\|_{H_{0}^{m}(\Omega)} .
$$

Proof. This follows from Theorem 3.1 and (4.4). 


\section{APPLICATION TO ESTIMATES FOR MULTILEVEL METHODS}

We will follow the notation in [1] and give a proof that (1.1) is satisfied in the case that $\Omega$ is a polygonal domain whose boundary is Lipschitz (i.e., all angles are less than $2 \pi$ ), the spaces $M_{k}$ are nested and defined by conforming piecewise polynomials. That is, $M_{k-1} \subset M_{k}$ and $M_{J} \subset H_{0}^{m}(\Omega)$. We assume that the initial triangulation is fixed independently of $J$ and gives rise to the others by means of a halving strategy. The form $A(\cdot, \cdot)$ is the standard generalized Dirichlet form of order $m$. Finally, we suppose that (4.2) is satisfied with $S_{h_{k}}=M_{k}$ and $h_{k}=h_{0} 2^{-k}$, the mesh size associated with $M_{k}$ and, for simplicity of notation, write $P_{k}$ for $P_{h_{k}}$.

We will prove the following theorem.

Theorem 5.1. There exists $C>0$ such that

$$
\sum_{k=1}^{J} \lambda_{k}\left\|\left(Q_{k}-Q_{k-1}\right) v\right\|_{L^{2}(\Omega)}^{2} \leq C\|v\|_{H_{0}^{m}(\Omega)}^{2} \quad \text { for all } v \in M_{J},
$$

where $Q_{k}$ is the $L^{2}(\Omega)$ orthogonal projection onto $M_{k}$ and $Q_{0}=0$.

Proof. The proof of this theorem is similar to that given in [2]. We note that $h_{k}^{2 m} \leq C \lambda_{k}^{-1}$. Using standard properties of $Q_{k}$ and the fact that $Q_{k}=$ $Q_{k-1} Q_{k}=Q_{k} Q_{k-1}$, we see that

$$
\sum_{k=1}^{J} \lambda_{k}\left\|\left(Q_{k}-Q_{k-1}\right) v\right\|_{L^{2}(\Omega)}^{2} \leq C \sum_{k=1}^{J} h_{k}^{-2}\left\|\left(Q_{k}-Q_{k-1}\right) v\right\|_{H_{0}^{m-1}(\Omega)}^{2} .
$$

By techniques similar to those of [4] it follows that $\left\|Q_{k} u\right\|_{H_{0}^{m-1}(\Omega)} \leq C\|u\|_{H_{0}^{m-1}(\Omega)}$. Hence, using this and the fact that $\left(Q_{k}-Q_{k-1}\right) v=0$ for $v \in M_{l}$ with $l<k$,

$$
\begin{aligned}
& \sum_{k=1}^{J} h_{k}^{-2}\left\|\left(Q_{k}-Q_{k-1}\right) v\right\|_{H_{0}^{m-1}(\Omega)}^{2}=\sum_{k=1}^{J} h_{k}^{-2}\left\|\left(Q_{k}-Q_{k-1}\right) \sum_{l=k}^{J}\left(P_{k}-P_{k-1}\right) v\right\|_{H_{0}^{m-1}(\Omega)}^{2} \\
& \quad \leq C \sum_{k=1}^{J}\left(\sum_{l=k}^{J}\left(h_{l} / h_{k}\right) h_{l}^{-1}\left\|\left(P_{l}-P_{l-1}\right) v\right\|_{H_{0}^{m-1}(\Omega)}\right)^{2} \\
& \quad=C \sum_{k=1}^{J}\left(\sum_{l=k}^{J}\left(h_{l} / h_{k}\right) h_{l}^{-1}\left\|\left(I-P_{l-1}\right)\left(P_{l}-P_{l-1}\right) v\right\|_{H_{0}^{m-1}(\Omega)}\right)^{2} \\
& \quad \leq C \sum_{k=1}^{J}\left(\sum_{l=k}^{J}\left(h_{l} / h_{k}\right)\left\|\left(P_{l}-P_{l-1}\right) v\right\|_{H_{0}^{m}(\Omega)}\right)^{2} .
\end{aligned}
$$

In the last inequality we used the regularity pickup error estimate of Theorem 4.2. Defining $E$ to be the symmetric $J \times J$ matrix with entries $E_{k l}=h_{l} / h_{k}=$ $2^{k-l}$ for $l \geq k$, and $\vec{\alpha}$ to be the vector with components $\left\|\left(P_{l}-P_{l-1}\right) v\right\|_{H_{0}^{m}(\Omega)}$, we see that

$$
\sum_{k=1}^{J}\left(\sum_{l=k}^{J}\left(h_{l} / h_{k}\right)\left\|\left(P_{l}-P_{l-1}\right) v\right\|_{H_{0}^{m}(\Omega)}\right)^{2} \leq|E \vec{\alpha}|^{2} .
$$

Since the row sums are uniformly bounded in $J$, the eigenvalues of $E$ are 
bounded and hence

$$
\begin{aligned}
|E \vec{\alpha}|^{2} \leq C|\vec{\alpha}|^{2} & \leq C \sum_{k=1}^{J} \mathscr{A}\left(\left(P_{l}-P_{l-1}\right) v,\left(P_{l}-P_{l-1}\right) v\right) \\
& =C \sum_{k=1}^{J} \mathscr{A}\left(\left(P_{l}-P_{l-1}\right) v, v\right)=C \mathscr{A}(v, v) \leq C\|v\|_{H_{0}^{m}(\Omega)}^{2} .
\end{aligned}
$$

This proves the theorem.

\section{BIBLIOGRAPHY}

1. J. H. Bramble, Multigrid methods, Pitman Research Notes in Mathematics Series, Longman Scientific \& Technical, London. Copublished with John Wiley \& Sons, Inc., New York, 1993.

2. J.H. Bramble and J.E. Pasciak, New estimates for multigrid algorithms including the V-cycle, Math. Comp. 60 (1993), 447-471.

3. J.H. Bramble, J.E. Pasciak, and J. Xu, Parallel multilevel preconditioners, Math. Comp. 55 (1990), 1-22.

4. J.H. Bramble and J. Xu, Some estimates for weighted $L^{2}$ projections, Math. Comp. 56 (1991), 463-476.

5. P.G. Ciarlet, The finite element method for elliptic problems, North-Holland, New York, 1978.

6. P.G. Ciarlet, Basic error estimates for elliptic problems, Finite Element Methods: Handbook of Numerical Analysis (P.G. Ciarlet and J.L. Lions, eds.), vol. II, North-Holland, New York, 1991, pp. 18-352.

7. P. Grisvard, Elliptic problems in nonsmooth domains, Pitman, Boston, 1985.

8. J.L. Lions and E. Magenes, Problèmes aux limites non homogènes et applications, Vol. 1, Dunod, Paris, 1968.

9. J.L. Lions and J. Peetre, Sur une classe d'espaces d'interpolation, Inst. Hautes Études Sci. Publ. Math. 19 (1964), 5-68.

10. P. Oswald, On discrete norm estimates related to multilevel preconditioners in the finite element method, Constructive Theory of Functions, Proc. Internat. Conf. Varna 1991 (K. G. Ivanov, P. Petrushev and B. Sendov, eds.), Bulgar. Acad. Sci., Sofia, 1992, pp. 203214.

11. J.-L. Zolesio, Interpolation d'espaces de Sobolev avec conditions aux limites de type mêlé, C.R. Acad. Sci. Paris 285 (1977), 621-624.

Department of Mathematics, Cornell University, Ithaca, New York 14853

Department of Mathematics, Texas A\&M University, College Station, TeXas 77843

E-mail address: bramble@math.tamu.edu 